\title{
MEFFORTMOB: A EFFORT SIZE MEASUREMENT FOR MOBILE APPLICATION DEVELOPMENT
}

\author{
Laudson Silva de Souza ${ }^{1}$ and Gibeon Soares de Aquino Jr. ${ }^{1}$ \\ ${ }^{1}$ Department of Informatics and Applied Mathematics, \\ Federal University of Rio Grande do Norte, Natal, Brazil
}

\begin{abstract}
The rise of the use of mobile technologies in the world, such as smartphones and tablets, connected to mobile networks is changing old habits and creating new ways for the society to access information and interact with computer systems. Thus, traditional information systems are undergoing a process of adaptation to this new computing context. However, it is important to note that the characteristics of this new context are different. There are new features and, thereafter, new possibilities, as well as restrictions that did not exist before. Finally, the systems developed for this environment have different requirements and characteristics than the traditional information systems. For this reason, there is the need to reassess the current knowledge about the processes of planning and building for the development of systems in this new environment. One area in particular that demands such adaptation is software estimation. The estimation processes, in general, are based on characteristics of the systems, trying to quantify the complexity of implementing them. Hence, the main objective of this paper is to present a proposal for an estimation model for mobile applications, as well as discuss the applicability of traditional estimation models for the purpose of developing systems in the context of mobile computing. Hence, the main objective of this paper is to present an effort estimation model for mobile applications.
\end{abstract}

\section{KEYWORDS}

Software Engineering, Software Quality, Estimating Software, Systematic Review, Mobile Applications, Mobile Computing

\section{INTRODUCTION}

The ITU (International Telecommunication Union) estimates that there are more than 6 (six) billion mobile clients worldwide. According to Gartner, 1.75 billion people own mobile phones with advanced capabilities; he also foresees further growth in the use of this technology in the upcoming years [Error! Reference source not found.]. There is a global trend towards the increase of the number of users connected to the network via mobile devices which, consequently, will create an increasing demand for information, applications and content for such equipments. New ways to use existing information systems are emerging. In particular, systems that were once accessed via web interfaces through personal computers physically located in offices, universities or homes are providing new ways to access from mobile devices which, in turn, have different requirements and capabilities than the personal computers.

Thus, we realize that traditional information systems are undergoing a process of adaptation to this new computing context. Current developments, including the increase of the computational power of these new devices, in addition to the integration of multiple devices on a single one and lined up with the change of the users' behavior, actually create a new environment for the development of computing solutions. However, it is important to note that the characteristics of

DOI : $10.5121 /$ ijsea.2014.5405 
International Journal of Software Engineering \& Applications (IJSEA), Vol.5, No.4, July 2014

this new context are different. They present new resources and, thereafter, new possibilities [Error! Reference source not found.], [Error! Reference source not found.], [Error! Reference source not found.] and [Error! Reference source not found.], as well as introduce non-existing restrictions in conventional systems [Error! Reference source not found.] and [Error! Reference source not found.].

The fact is that this new technological scenario that is emerging with new requirements and restrictions requires a reevaluation of current knowledge about the processes of planning and building software systems. These new systems have different characteristics and, therefore, an area in particular that demands such adaptation is software estimation. The estimation processes, in general, are based on characteristics of the systems, trying to quantify the complexity of implementing them. For this reason, it is important to analyze the methods currently proposed for software projects estimation and evaluate their applicability to this new context of mobile computing.

Hence, the main objective of this paper is to present a proposal for an estimation model for mobile applications, as well as discuss the applicability of traditional models used in estimation of information systems for the purpose of the development of systems in the context of mobile computing. In this work, the main estimation methods that exist now will be analyzed, the specific characteristics of mobile systems will be identified and an adaptation of a estimation method that exists in this context will be proposed.

\section{ESTIMATION METHODS}

In order to identify how the traditional estimation methods could address the characteristics of the systems, a literature review on the main estimation methods was performed. The methods identified in the survey can be seen in Table 1 . 
Table 1. Main Estimation Methods.

\begin{tabular}{|c|c|c|}
\hline Year & Method & Author \\
\hline 1979 & Function Point Analysis (FPA) & Albrecht [11] \\
\hline 1981 & COnstructive COst MOdel (COCOMO) & Barry W. Boehm 's [12] \\
\hline 1982 & DeMarco's Bang Metrics & Tom DeMarco [13] \\
\hline 1986 & Feature Points & Jones [14] \\
\hline 1988 & Mark II FPA & Charles Symons [14] \\
\hline 1989 & Data Points & Harry Sneed [15] \\
\hline 1990 & $\begin{array}{l}\text { Netherlands Software Metrics Users Association } \\
\text { (NESMA) FPA }\end{array}$ & $\begin{array}{l}\text { The Netherlands Software Metrics } \\
\text { Users Association [16] }\end{array}$ \\
\hline 1990 & $\begin{array}{l}\text { Analytical Software Size Estimation Technique- } \\
\text { Real-Time (ASSET-R) }\end{array}$ & Reifer [17] \\
\hline 1992 & 3-D Function Points & Whitmire [18] \\
\hline 1993 & Use Case Points UCP & Gustav Karner [19] \\
\hline 1994 & Object Points & Banker et al. [20] \\
\hline 1994 & $\begin{array}{l}\text { Function Points by Matson, Barret and } \\
\text { Mellichamp }\end{array}$ & Matson, Barret e Mellichamp [21] \\
\hline 1997 & Full Function Points (FFP) & $\begin{array}{l}\text { University of Quebec in } \\
\text { cooperation with the Software } \\
\text { Engineering Laboratory in Applied } \\
\text { Metrics [18] }\end{array}$ \\
\hline 1997 & Early FPA (EFPA) & Meli, Conte et al. [22] \\
\hline 1998 & Object Oriented Function Points - (OOFPs) & Caldiera et al. [23] \\
\hline 1999 & Predictive Object Points - (POPs) & Teologlou [24] \\
\hline 1999 & $\begin{array}{l}\text { Common Software Measurement International } \\
\text { Consortium (COSMIC) FFP }\end{array}$ & $\begin{array}{l}\text { Common Software Measurement } \\
\text { International Consortium } \\
\text { (COSMIC) [25] }\end{array}$ \\
\hline 2000 & $\begin{array}{l}\text { Early \& Quick COSMIC-Full Function Points } \\
\text { (E\&Q COSMIC FFP) }\end{array}$ & Meli et al. [26] \\
\hline 2000 & Kammelar's Component Object Points & Kammelar [27] \\
\hline 2001 & $\begin{array}{l}\text { Object Oriented Method Function Points - } \\
(\text { OOmFP })\end{array}$ & Pastor and his colleagues [28] \\
\hline 2004 & Finnish Software Metrics Association FSM & $\begin{array}{l}\text { The Finnish Software Metrics } \\
\text { Association (FiSMA) [29] }\end{array}$ \\
\hline
\end{tabular}

Table 1 displays in chronological order the main estimation methods, showing the year of creation, the name of the method and the author of it. At first glance, one realizes that the main existing methods were not designed to consider the requirements of mobile applications. Indeed, the very creation of most of them precedes the emergence of mobile devices as we know today. This suggests that the use of these methods to estimate the effort of the development of projects involving systems or applications for mobile devices would cause a possible failure to quantify the complexity of some features and, therefore, would not produce adequate estimates.

\section{Characteristics of Mobile Applications}


International Journal of Software Engineering \& Applications (IJSEA), Vol.5, No.4, July 2014

In order to identify characteristics that are inherent to systems and mobile applications, a surveying of the characteristics of these types of software was accomplished through a systematic review. Conducting a systematic review is relevant because most searches begin with some kind of review of the literature, and a systematic review summarizes the existing work fairly, without inclinations. So the surveys were conducted according to a predefined search strategy, in which the search strategy should allow the integrity of the research to be evaluated. The planning and accomplishment of the methodology discussed were directed by Procedures for Performing Systematic Reviews [Error! Reference source not found.].

\subsection{Planning The Systematic Review}

In the context of research questions, the following research question was formulated: "What are the characteristics of Mobile Applications?", based on the issue about the proposed study.

Search Strategies - The search strategy was divided into three parts: sources, keywords and search strings.

Sources - the researches were directed to the following databases:

- ACM DL Digital Library (http://dl.acm.org/)

- Google Scholar (http://scholar.google.com.br/)

- IEEE Xplore Digital Library (http://ieeexplore.ieee.org/Xplore/).

Keywords - the keywords were defined and based on the research question elicited previously and on their synonyms, as follows:

- Mobile; Applications; Computing; Features; Characteristics; Attribute; Aspect; Property; Factors; Individuality; Differential; Detail; Software; System;

earch string - based on the keywords defined previously and according to the sources to be used, the following search string was prepared:

- “(("Mobile Applications”) OR ("Mobile Computing”) OR ("Mobile System”) OR ("Mobile Software")) AND (Features OR Characteristics OR Attribute OR Aspect OR Property OR Factors OR Individuality OR Differential OR Detail)".

The results obtained through the researches made with the string search string defined previously in the three databases mentioned above were analyzed according to the following criteria:

- Inclusion Criteria:

- The returned result should be available in English or Portuguese;

- The returned result should be available in PDF or HTML format;

- The returned result should answer the research question directly;

- Exclusion Criteria:

- The returned result has already been found in previous research;

- The returned result has not been published in conferences, books, newspapers or magazines;

- The returned result has no relation to the research question;

$\circ$ The access to the result is not available through agreements with CAPES or UFRN; 
○ The returned result was not published between 2002 and 2013;

Procedures for The Evaluation of the Articles: the articles will be analyzed considering its relation with the issues addressed in the research questions, inclusion criteria and exclusion criteria, and their respective situation will be assigned with either "Accepted" or "Rejected". The evaluation will follow the following procedure: read the title and abstract and, should it be related with the research question, also read the whole article.

\subsection{Implementation of the Systematic Review}

The implementation of the systematic review was performed almost in line with its planning, except for the need to adjust the syntax of the proposed search string due to the particularities of the research bases. 234 articles were analyzed, of which 40 were selected and considered "Accepted" according to the inclusion criteria; 194 were considered "Rejected" according to the exclusion criteria. The list with all the articles can be accessed at the following address: http://www.laudson.com/sr-articles.pdf. The 40 articles that were accepted were fully read, thus performing the data extraction. All the characteristics found during this extraction phase were described in the following subsection.

\subsection{Completion of Systematic Review}

Given the results extracted from the systematic review, it's is possible to identify 29 kinds of characteristics in $100 \%$ of the articles evaluated and considered accepted in accordance with the inclusion criteria. However some of these are a mixture of characteristics of mobile devices and characteristics of mobile applications, such as the characteristic called "Limited Energy", which is a characteristic of the device and not the application, however the articles that mention this type of characteristic emphasize that in the development of a mobile application, this "limitation" must be taken into account since all the mobile devices are powered by batteries, which have a limited life, depending completely on what the user operates daily. Applications requiring more hardware or software resources will consume more energy. In Figure 1, the 23 types of characteristics mentioned the most in the selected articles can be observed. 


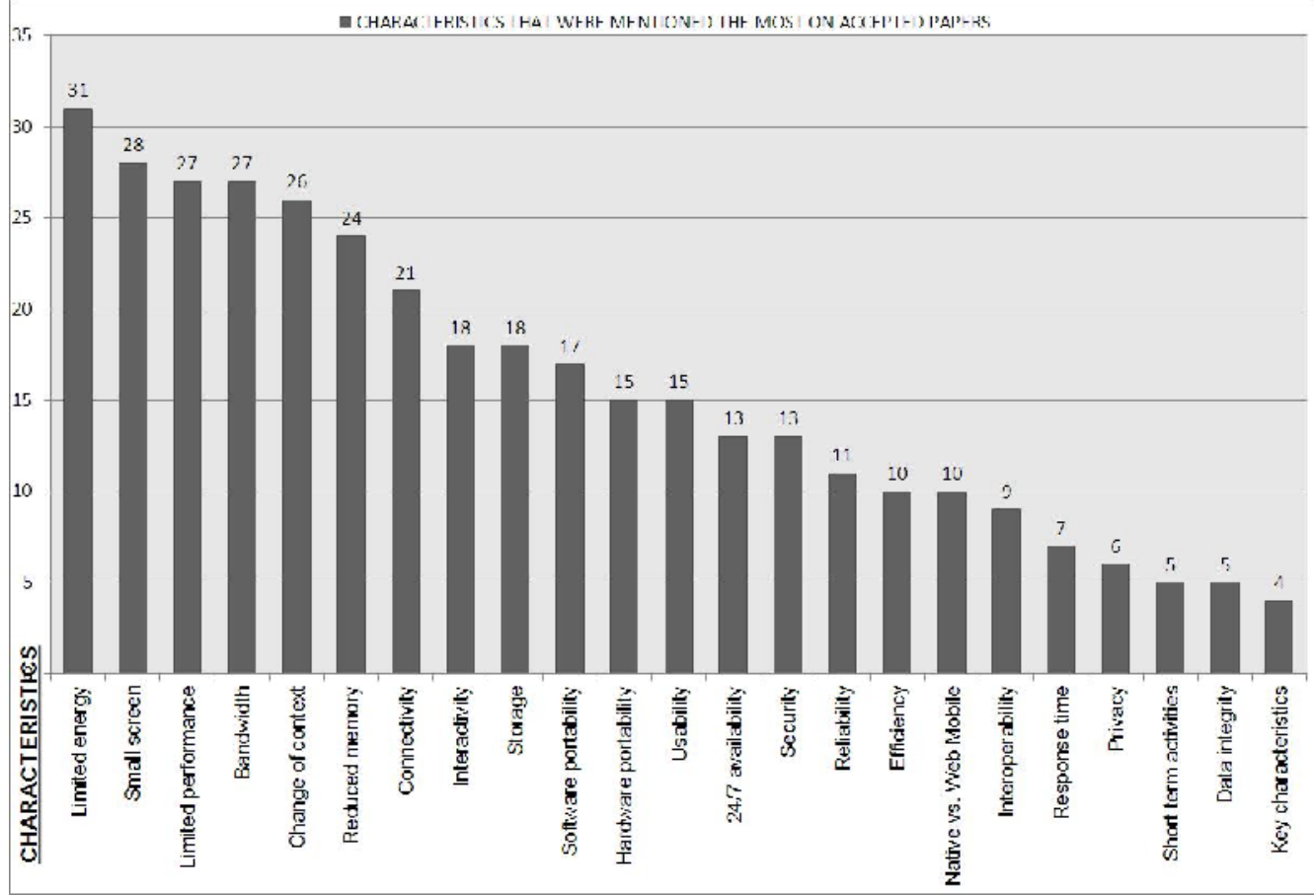

Figure 1. Characteristics that were mentioned the most on accepted papers

The other six types of characteristics identified are mentioned only three times, which are: "Complex integration of tasks in real time" and "Constant interruption of activities"; and, finally, are mentioned only once, which are: "Functional area", "Price", "Target public" and "Type of provider Type".

Following, there is a description of each characteristic identified in the review:

- Limited energy: every mobile device is powered by battery and, because of this, it has a certain lifetime period [Error! Reference source not found.].

- Small screen: mobile device screens are pretty small and, because of this, interface design is limited [Error! Reference source not found.].

- Limited performance: due to its size and technological advancement all mobile devices, even the most advanced in its class, have limitations of specific resources such as processing power, memory and connectivity. Because of this, the performance is limited [Error! Reference source not found.].

- Bandwidth: given an application that requires the maximum, the minimum or a reasonable bandwidth, one must consider its enormous variation [Error! Reference source not found.].

- Change of context: the change of context occurs in accordance with the environment [Error! Reference source not found.].

- Reduced memory: due to its size and technological advancement, all mobile devices, even the most advanced in its class, have limitations of specific resources, including the size of its memory [Error! Reference source not found.].

- Connectivity: the kind of connectivity that the application will use, such as 3G, bluetooth, infrared and Wi-Fi [Error! Reference source not found.].

- Interactivity: what will be the type of input that the user will use to interact with the application [Error! Reference source not found.]. 
- Storage: the applications have to take into consideration how it is going to be done [Error! Reference source not found.].

- Software portability: the application should be performed on all types of operating systems [Error! Reference source not found.].

- Hardware portability: the application should be performed on all types of devices [Error! Reference source not found.].

- Usability: is a set of attributes which affect the effort needed for the use, in which it must be intuitive and as natural as possible to make or receive a call or text message [Error! Reference source not found.].

- 24/7 availability: the application must be available to access anywhere, anytime [Error! Reference source not found.].

- Security: must prevent accidental or deliberate unauthorized access to the applications and data [Error! Reference source not found.].

- Reliability: is a set of attributes which affect the application's ability to maintain its level of performance under stated conditions for a stated period of time [Error! Reference source not found.].

- Efficiency: is a set of attributes that relate to the relationship between the application's level of performance and the amount of resources used, under stated conditions [Error! Reference source not found.].

- Native vs. Web Mobile: it must be defined if the application will be designed to be installed on the device itself, which is known as native applications, or used on the web [Error! Reference source not found.].

- Interoperability: the application should be able to interact with other specific systems. In other words, it must have interoperability with other services [Error! Reference source not found.].

- Response time: the applications must be initialized and finalized immediately [Error! Reference source not found.].

- Privacy: the application must demonstrate to the user how his or her personal information are being collected, used and shared, and let the user exercise his or her choice and control over their use [Error! Reference source not found.].

- Short term activities: activities in mobile applications tend to have a short duration, ranging from several seconds to several minutes [Error! Reference source not found.].

- Data integrity: making sure that in an accidental shutdown of the application or of the device itself, the application will ensures data integrity [Error! Reference source not found.].

- Key characteristics: mobile applications tend to be more focused or, in other words, they have specific key characteristics rather than offer the exploratory environment commonly used [Error! Reference source not found.].

- Complex integration of real-time tasks: mobile applications should provide integration between application of different sources (native or web) [Error! Reference source not found.].

- Constant interruption of activities: when using a mobile application, the activities are constantly interrupted, like when you receive a call, lose connection or have a low battery, which are examples of such interruptions [Error! Reference source not found.].

- Functional area: data, collaboration and communication services, information services and productivity services such as business and office applications [Error! Reference source not found.].

- Price: free, less than five euros and more than five euros [Error! Reference source not found.].

- Target audience: applications for final private consumer or business applications [Error! Reference source not found.]. 
International Journal of Software Engineering \& Applications (IJSEA), Vol.5, No.4, July 2014

- Provider type: businesses, professionals or other service providers [Error! Reference source not found.].

After this survey, a refinement was made and a mix of characteristics was elicited with the purpose of defining which characteristics would be emphasized. Of a total of 23 types of characteristics that were most mentioned in the selected articles, a common denominator of 13 characteristics was reached, some of which had their names redefined, like "Interactivity", which became "Input Interface".

\section{Characteristics of Mobile Applications Survey}

With the conclusion of the systematic review, a survey was carried out among experts in mobile development with the purpose of ratifying the characteristics previously raised and to prove their respective influence on mobile development. The disclosure of the survey was conducted in more than 70 locations, among them universities and businesses, through e-mails, study groups and social groups.

In general, of all 117 feedbacks received through the survey, $100 \%$ of the experts confirmed the characteristics; among them, an average of $72 \%$ indicated a greater effort and complexity regarding the characteristics during development, an average of $12 \%$ indicated less effort and complexity and, finally, an average of $16 \%$ indicated they did not perceive any difference in mobile development, even though they confirmed the presence of the characteristics.

\section{Problem AdDresSed}

As noted in Section II, there is no estimation method developed for mobile applications projects. Moreover, some of the characteristics elicited in Section III aggravate the complexity and, thereafter, the effort in the development of mobile applications.

From the analysis that follows, with the characteristics of applications on mobile devices elicited in Section III, it is clear that they are different from the characteristics of traditional systems and directly influence its development. A clear example, which is different from the information or desktop systems, is the characteristic that the mobile devices have "Limited Energy". As mobile devices are powered by battery, which have a limited lifetime period, the applications must be programmed to require the minimal amount of hardware resources possible, since the more resources consumed, the greater amount of energy expended. This characteristic makes it necessary for the solution project to address this concern, generating a higher complexity of development and, thereafter, a greater effort and cost.

Another specific characteristic of this context is the "Graphical Interface". Due to the reduced screen size, the interface design is limited. Therefore, a greater complexity and, thereafter, a larger effort is required in the development of the graphical interface. Another characteristic related to the screen is the "Input Interface", which defines how the user will interact with the application, in other words, if the user will interact via keypad, stylus, touch screen or voice and image recognition. The latter makes the task of developing applications that offers all these interaction options more complex, thus requiring a bigger effort.

Regarding connectivity, the characteristic "Bandwidth" was identified, wherein a mobile application might have the maximum band at times and the minimum in other moments. Some types of applications need to realize this and act differently in each situation. Another related feature is the "Connectivity Type". Mobile applications can be developed to support different types of connectivity such as $3 \mathrm{G}$, bluetooth, infrared, Wi-Fi, Wireless, NFC and others. In 
International Journal of Software Engineering \& Applications (IJSEA), Vol.5, No.4, July 2014

addition, a single application can support multiple types of connectivity simultaneously. These behaviors directly affect the complexity of the software and therefore require a larger development effort.

The "Change in Context" is also another characteristic inherent in mobile applications, which should take into account not only the data entries explicitly provided by users, but also the implicit entries concerning the physical and computational context of the users and the environments that surround them. In addition, the "Constant Interruption of Activities" is a much more common characteristic in this context, as well as the need for some applications to be developed to work offline and therefore be able to synchronize. Mobile applications should be prepared for different scenarios because the activities are interrupted constantly. Receiving a call, lack of connection and low battery are examples of such interruptions, which makes the applications become much more complex.

Despite the advances related to the computational ability of these devices, their hardware must still be considered as limited, especially when compared to desktops and servers. Two characteristics related to this issue are "Limited Performance" and "Reduced Memory". Besides these, a characteristic inherent to the use of mobile devices is the "Response Time", that is directly related to the power of "Processing". Mobile applications must be initialized and finalized immediately, in other words, any development should be focused in the time variable. These characteristics require the applications to be developed with a possible resource optimization for a better efficiency and response time, requiring more effort.

The "Portability" is also a required characteristic of these applications. It can be divided into two characteristics: the "Hardware Portability" and the "Software Portability". Regarding the first one, nowadays there is a large number of different devices with different capabilities and resources. A mobile application should be able to run on the largest number of devices possible. This requires an increased effort in the development. Moreover, a greater effort in testing this kind of portability is required. Regarding "Software Portability", it is necessary to develop specific applications for each existing platform should the application be native. With this, more effort is required for replications of the same software product, including the tests.

Finally, mobile applications can be separated into two types: "Native or Web Mobile". The first one has higher performance and easiness in accessing the hardware, while the second has lower performance since it is web, but it is easier to achieve portability. In addition, there are some applications that are considered hybrids. Depending on the type of application, the issues that must be considered and the complexity can be different, requiring different development efforts.

From the survey of the most popular estimation methods cited in Section III, it was found that these characteristics are not covered by the current estimation methods for two explicit reasons: first, none of the existing methods was designed to perform project estimation in mobile applications development; and second, all the characteristics discussed in this section are exclusive to mobile applications, with direct interference in their development, thereby generating a greater complexity and, thereafter, a greater effort. However, to consider any of the existing estimation methods to apply to the process of development of mobile applications is to assume that this kind of development is no different than the project of developing desktop applications, in other words, an eminent risk is assumed.

\section{Proposal: Estimation in Mobile Application Development Project}


A solution to solve this problem would be to create a new estimation method or even to adapt some existing estimation method, in which would be added all of the characteristics identified that directly affect the mobile application development project, taking into account whatever is needed to reach the minimum efficiency in the estimates.

The approached proposed is an adaptation of an existing method, based exclusively on methods recognized as international standards by ISO. Among the most popular estimation methods mentioned in Section III, the method used to base the proposal below on is known as "Finnish Software Metrics Association (FiSMA)". The model is one of the five methods for measuring software that complies with the ISO/IEC 14143-1 standard, is accepted as an international standard for software measuring [Error! Reference source not found.] and nowadays over 750 software projects are completed being estimated by FISMA. However, the difference between this and other methods that are in accordance with the above standard, which are the Common Software Measurement International Consortium Function Points (COSMIC FP) [Error! Reference source not found.], the International Function Point Users Group (IFPUG) FPA [Error! Reference source not found.], MarkII FPA [Error! Reference source not found.] and the Netherlands Software Metrics Association (NESMA) WSF [Error! Reference source not found.], is that the method used is based in functionality but is service-oriented. It also proposes in its definition that it can be applied to all types of software, but this statement is lightly wrong since in its application, the method does not take into account the characteristics elicited in Section IV.

The COMISC FP [Error! Reference source not found.], the MarkII FPA [Error! Reference source not found.] and the NESMA [Error! Reference source not found.] were created based on the FPA [Error! Reference source not found.], in other words, they assume the counting of Function Point (FP), but considering the implemented functionality from the user's point of view. With this, it is clear that the methods mentioned above do not take into account the characteristics of mobile applications because they are not noticed by the user. The methods are independent of the programming language or technology used. And, unlike FISMA, they do not bring in their literature the information that they can be applied to all types of software.

Overall, the FISMA method proposes that all services provided by the application are identified. It previously defines some services, among which stands out the user's interactive navigation, consulting services, user input interactive services, interface services for other applications, data storage services, algorithmic services and handling services. Finally, after identifying all the services, the size of each service is calculated using the same method and thus obtaining a total functional size of the application by adding the size of each service found [Error! Reference source not found.].

\subsection{Approaching the Chosen Model}

The FiSMA method in its original usage proposes a structure of seven classes of the Base Functional Component or BFC (Base Functional Component) type, which is defined as a basic component of functional requirement. The seven classes used to account for the services during the application of the method are [Error! Reference source not found.]:

- Interactive navigation of the end user and query services (q):

- specify all parts of the interactive user interface where there is no maintenance of persistent data storage(s) of the system. Maintenance refers to any service where data is changed as a result of the service and includes creating, updating or deleting. Amount of size units of navigation and query functions depends on the 
number of different data items of the BFC measured and the number of needed reading references to entity types.

- Interactive input services from end users (i):

- specify all parts of the interactive user interface where there is maintenance of persistent data storage(s) of the system. Data storages consist of entities (data records). Maintaining refers to any service where data is changed as a result of the service and includes creating, updating and deleting. From a user's point of view, interactive end-user services are used to perform those business tasks which change the data contents of the system. From the information system point of view end-users manipulate system data using interactive end-user services. Amount of size units of input functions depends on the number of different data items of the BFC measured, the number of needed reading references and the number of needed writing references to entity types.

- Non-interactive outbound services for the end user (o):

- specify all parts of the user interface which are non-interactive and do not maintain persistent data storage(s) of the system. Amount of size units of output functions depends on the number of different data items of the BFC measured and the number of needed reading references to entity types.

- Interface services for another application $(\mathrm{t})$ :

- specify all automatized data transfer functions moving data groups from the measured piece of software to any other application or any device. Amount of size units of outbound interface functions depends on the number of different data items of the BFC measured.

- Interface services for other applications (f):

- specify all automatized data transfer functions, receiving data groups provided and sent by any other application or any device. Amount of size units of inbound interface functions depends on the number of different data items of the BFC measured, the number of reading references and the number of writing references to entities.

- Data storage services (d):

- specify a group or collection of related and self-contained data in the real world about which the user requires the software to provide persistent storage. Data storage services are functional services provided by the piece of software to satisfy these data storage requirements. These "groups or collections of related and self-contained data" are often called as entity types, data groups, data classes or objects of interest, depending on the terminology used in the development environment. Data storage services store data persistently and make it available for maintenance, inquiry, or output. Data storage services are typically implemented as tables in relational databases, or as records in data fi les in general. Amount of size units of data storage services depends on the number of different data items i.e. the number of attributes related together in the selfcontained group or collection.

- Algorithmic manipulation services (a):

- Definition of BFC class: Algorithms are user-defined, independent data manipulation routines. Independence means here that the functionality of the routine is not included in the normal functionality of any function of any other BFC type. Algorithmic manipulation may consist of arithmetic and/or logical operations. Amount of size units of algorithmic and manipulation services depends on the number of different operations performed and the number of different variables needed. 
International Journal of Software Engineering \& Applications (IJSEA), Vol.5, No.4, July 2014

The identification for each class name BFC previously mentioned, with a letter in parenthesis, is used to facilitate the application of the method during the counting process, because each of the seven classes BFCs are composed of other BFC classes which, at the time of calculating, these BFCs "daughter" classes are identified by the letter of their BFC "mother" class followed by a numeral, as can be seen in Figure 2.

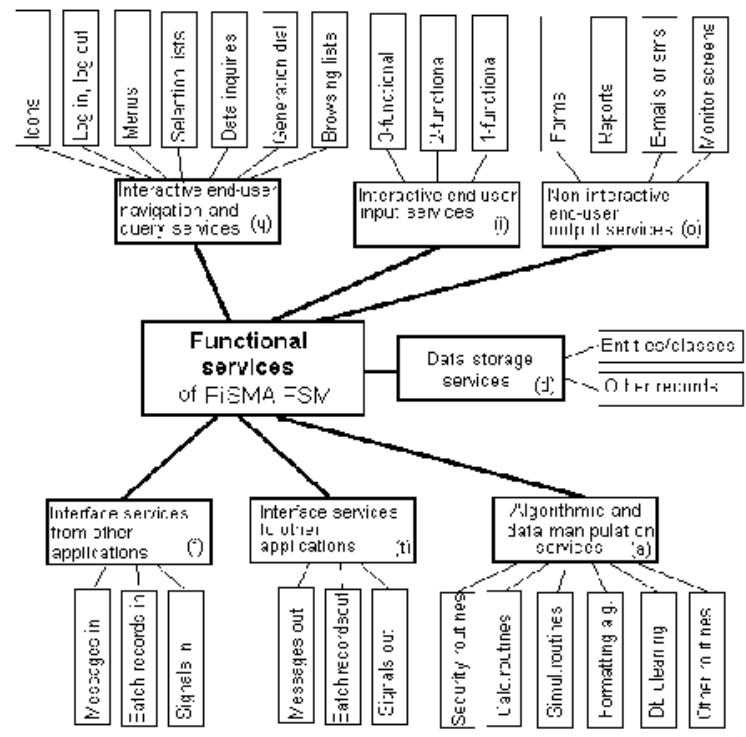

Figure 2. Types of BFCs classes of the base model [Error! Reference source not found.]

The unit of measurement is the point of function with the letter " $F$ " added to its nomenclature to identify the "FiSMA", resulting in FfP (FiSMA Function Point) or Ffsu (FiSMA functional size unit). The measurement process generally consists of measuring the services and end-user interface and the services considered indirect [Error! Reference source not found.], as can be seen in Figure 3.

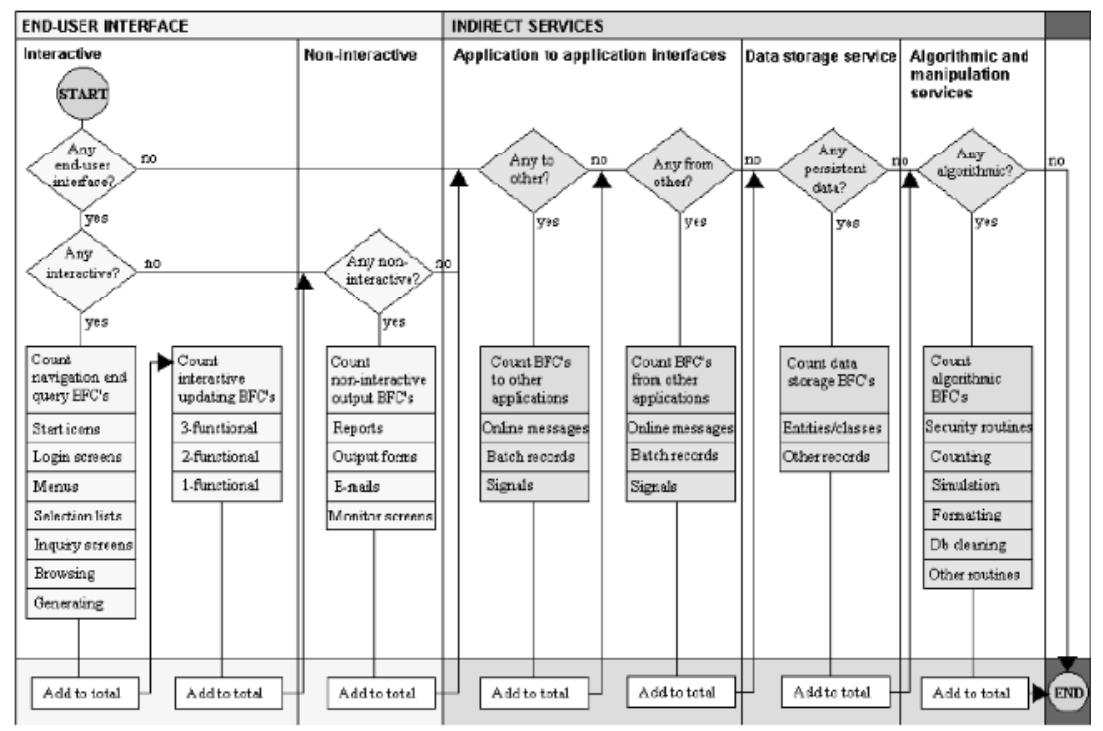


Figure 3. Representation of the measurement process of the base model [Error! Reference source not found.]

Figure 3 shows the process of measuring the base model, in which it defines each step and sum of each BFC class of the model. Briefly, the process of counting should be done as follows. Identify: 1-How many types of BFCs does the software have? 2-Which are they? (identify all) 3-What are they? (provide details of each BFC identified)

After doing this, it is necessary to add each BFC root using the formulas pre-defined by the method and their assignments. Finally, the formula of the final result of the sum is the general sum of all the BFCs classes.

\subsection{Applying the Chosen Model}

The FiSMA method can be applied manually or with the aid of the Experience Service ${ }^{1}$ tool, which was the case, provided by FiSMA itself through contact made with senior consultant Pekka Forselius and with the chairman of the board Hannu Lappalainen.

When using the tool, it is necessary to perform all the steps of the previous subsection to obtain the functional size. Figure 4 shows the final report after the implementation of the FiSMA on a real system, the Management of Academic Activities Integrated System (Sigaa) in its Mobile version, developed by the Superintendence of Computing (SINFO) of the Federal University of Rio Grande do Norte (UFRN).

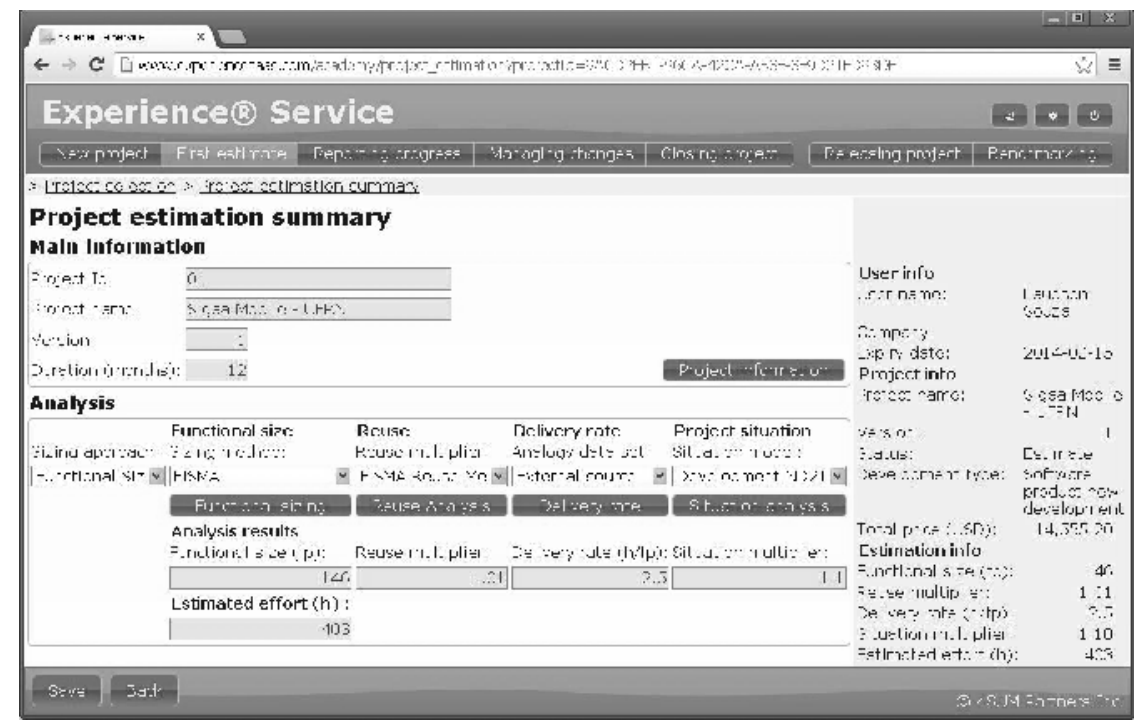

Figure 4. Final Report of FiSMA applied to Sigaa Mobile

After the application of FiSMA, the functional size of the software is obtained and from this it is possible to find the effort using the formula: Estimated effort $(\mathrm{h})=$ size (fp) $\mathrm{x}$ reuse $\mathrm{x}$ rate of delivery $(\mathrm{h} / \mathrm{fp}) \mathrm{x}$ project status; the latter is related to productivity factors that are taken into account for the calculation of the effort. However, of the factors predefined by the FiSMA regarding the product, only 6 (six) are proposed, in which the basic idea of the evaluation is that

\footnotetext{
${ }^{1}$ http://www.experiencesaas.com/
} 
"the better the circumstances of the project, the more positive the assessment". The weighting goes from - - to ++ , as follows:

\section{Caption:}

- " $++"=[1.10]$ Excellent situation, much better circumstances than in the average case

- " $+"=[1.05]$ Good situation, better circumstances than in the average case

- " + / -" = [1.0] Normal situation

- "-" = [0.95] Bad situation, worse circumstances than in the average case

- “- -" $=[0.90]$ Very bad situation, much worse circumstances than in the average case

\section{Productivity factors:}

Functionality requirements: compatibility with the needs of the end user, the complexity of the requirements.

- (- -) Complex and critical application area (thousands of FPs), multiple users and multicultural system.

- ( - ) Interoperable application area with some complex characteristics, requiring special understanding from users and developers.

- (+ / -) Partly automated, integrated application area and a medium size application (between 600 and 1000 FPs) with standard security requirements.

- (+ ) Application area mostly automated and application with less than 5 interfaces with other systems; there are specific security requirements.

- $(++)$ Very mature application area, simple and easy, a small stand-alone application (less than 200 FPs) for a small group of users.

Reliability requirements: maturity, tolerance to faults and recovery for different types of use cases.

- (- -) Malfunctions may put in danger human lives and cause significant economic or environmental losses. \}

- ( - ) The software is part of a large real-time system where all the failures of operation will cause problems to many other applications.\}

- (+ / -) Not more than 2 hours of downtime is acceptable, but the system recovery routines are appropriate.

- $\quad(+)$ Need for non-continuous operation, but daily.

- $(++)$ Need for periodic operation. Pausing for a few days will not cause any damage to the organization.

Usability requirements: understandability and easiness to learn the user interface and workflow logic.

- (- -) A large number of different types of end users around the world.

- ( - ) 2 or 3 different types of users with different skills.

- (+ / -) A large number of end users with equal abilities.

- $\quad+$ ) No more than tens or hundreds of homogeneous users in perhaps more than one location.

- (+ + ) Only a few users, all located on one site.

Efficiency requirements: effective use of resources and adequate performance in each use case and under a reasonable workload.

- (- -) Complex database with millions of data records and transactions per day, thousands of simultaneous end users.

- ( - ) Large database, hundreds of simultaneous end users, critical response most of the time.

- (+ / -) Large database, less than millions of data records and less than hundreds of simultaneous end users. 
International Journal of Software Engineering \& Applications (IJSEA), Vol.5, No.4, July 2014

- $(+)$ Medium database in volume and structure, simple and predictable data requests from some simultaneous end users.

- (+ +) Simple and small database without simultaneous end users or complex data requests.

Maintainability requirements: lifetime of the application, criticality of fault diagnosis and test performance.

- (- -) Very large strategic software (over 20 years of lifetime) in a volatile area of business, with frequent changes in laws, regulations and business rules.

- ( - ) Large software (10-20 years of lifetime), and frequent changes in laws, regulations and business rules.

- (+ / -) Medium size software (5-10 years of lifetime), monthly changes in laws, regulations and business rules.

- (+ ) Small software, rarely changes (2 to 5 years of lifetime).

- $(++)$ Temporary software (less than 2 years of lifetime), without modifications.

Portability requirements: adaptability and instability to different environments, to the architecture and to structural components.

- (- -) Software users are located in many types of organizations, with various platforms (hardware, browsers, operating systems, middleware, protocols, etc), various versions and various update frequencies.

- ( - ) The software must operate on some different platforms (hardware, browsers, operating systems, middleware, protocols, etc) and in various versions of each of them.

- (+ / -) Each version of the software must run on multiple versions of a given platform (hardware, browser, operating system, middleware, protocols, etc), and the frequencies of update of the users are quite predictable.

- $(+)$ The software must run on a given platform (hardware, browser, operating system, middleware, protocols, etc), but the use of system-level services is limited because the upgrade process is partial.

- (+ +) Software must be run on a particular platform (hardware, browser, operating system, middleware, protocols, etc), but the upgrade process is completely controllable.

Among the productivity factors mentioned above, only the "Portability Requirement" factor fits in harmony with the "Portability" characteristic regarding both hardware and software. However, none of the other factors discusses the characteristics of mobile application, in other words, after obtaining the functional size of the software and applying the productivity factors related to the product to estimate the effort, this estimate ignores all of the characteristics of mobile applications, judging that the estimate of traditional information systems is equal to the mobile application. However, with the proposal of the creation of new productivity factors, which would be the specific characteristics of mobile applications, this problem will be solved, as presented below.

\section{Performance Factor:}

- ( - ) The application should be concerned with the optimization of resources for a better efficiency and response time.

- (+ / -) Resource optimization for better efficiency and response time may or may not exist.

- (+ ) Resource optimization for better efficiency and response time should not be taken into consideration.

\section{Power Factor:}

- ( - ) The application should be concerned with the optimization of resources for a lower battery consumption. 
International Journal of Software Engineering \& Applications (IJSEA), Vol.5, No.4, July 2014

- (+ / -) Resource optimization for lower battery consumption may or may not exist.

- $(+)$ Resource optimization for a lower battery consumption should not be taken into consideration.

\section{Band Factor:}

- ( - ) The application shall require the maximum bandwidth.

- (+ / -) The application shall require reasonable bandwidth.

- $(+)$ The application shall require a minimum bandwidth.

\section{Connectivity Factor:}

- ( - ) The application must have the maximum willingness to use connections such as 3G, Wifi, Wireless, Bluetooth, Infrared and others.

- (+ / -) The application must have reasonable predisposition to use connections such as 3G, Wi-Fi and Wireless.

- ( + ) The application must have only a predisposition to use connections, which can be: 3G, Wi-fi, Wireless, Bluetooth, Infrared or others.

\section{Context Factor:}

- ( - ) The application should work offline and synchronize.

- (+ / -) The application should work offline and it is not necessary to synchronize.

- (+) The application should not work offline.

\section{Graphic Interface Factor:}

- ( - ) The application has limitations due to the screen size because it will be mainly used by cell phone users.

- (+ / -) The application has reasonable limitation due to the screen size because it will be used both by cell phone and tablet users.

- ( + ) The application has little limitation due to the screen size because it will be mainly used by tablet users.

\section{Input Interface Factor:}

- ( - ) The application must have input interfaces for touch screen, voice, video, keyboard and others.

- (+ / -) The application must have standard input interfaces for keyboard.

- ( + ) The application must have any one of the types of interfaces, such as: touch screen, voice, video, keyboard or others.

The proposed factors take into account the same weighting proposed by FiSMA, but only ranging from - to + , in other words:

- " $+"=[1.05]$ Good situation, better circumstances than in the average case

- " + / -" = [1.0] Normal Situation

- “-" $=[0.95]$ Bad situation, worse circumstances than in the average case

$\bullet$ 
International Journal of Software Engineering \& Applications (IJSEA), Vol.5, No.4, July 2014

The functional size remains the same, thus affecting only the formula used to obtain the effort, which will now consider in its "project situation" variable the new productivity factors specific for mobile applications.

\section{CONCLuSion}

Given the results presented, based on the literature review of estimation methods and on the systematic review of the characteristics of mobile applications, it was observed that this sub-area of software engineering still falls short. Basically, it's risky to use any existing estimation method in development projects for mobile applications, as much as there are some models already widespread in industry, such as the Function Point Analysis, the Mark II and the COSMIC-FFP, which are even approved by ISO as international standards. They all fall short by not taking into account the particularities of mobile applications, which makes the method partially ineffective in this situation.

With the common emergence of new systems, experts always find a barrier when using one of the current methods of software measurement. This barrier can be on the effectiveness of the method, on what type of method should be used, when it comes to a software that is considered unconventional and, mostly, when it is required to apply it in completely atypical scenarios. This whole situation is aggravated further when it comes to mobile applications.

Based on this study, it is concluded that the proposal presented in this work is entirely appropriate and viable and that this proposal should take into account all the peculiarities of such applications, finally creating a belief that there actually are considerable differences in the development project for mobile applications.

\section{REFERENCES}

[1] L. Naismith, M. Sharples, G. Vavoula, P. Lonsdale et al., "Literature review in mobile technologies and learning," 2004.

[2] G. Macario, M. Torchiano, and M. Violante, "An in-vehicle infotainment software architecture based on google android," in Industrial Embedded Systems, 2009. SIES '09. IEEE International Symposium on, 2009, pp. 257-260.

[3] T. Liu, H. Wang, J. Liang, T.-W. Chan, H. Ko, and J. Yang, "Wireless and mobile technologies to enhance teaching and learning," Journal of Computer Assisted Learning, vol. 19, no. 3, pp. 371-382, 2003.

[4] I. GARTNER. (2013) Gartner says worldwide mobile phone sales declined 1.7 percent in 2012.

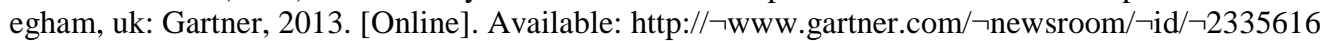

[5] C.-C. Yang, H.-W. Yang, and H.-C. Huang, "A robust and secure data transmission scheme based on identity-based cryptosystem for ad hoc networks," in Proceedings of the 6th International Wireless Communications and Mobile Computing Conference, ser. IWCMC '10. New York, NY, USA: ACM, 2010, pp. 1198-1202, aCM. [Online]. Available: http://ᄀdoi.acm.org/ $\neg 10.1145 / \neg 1815396.1815670$

[6] I. Ketykó, K. De Moor, T. De Pessemier, A. J. Verdejo, K. Vanhecke, W. Joseph, L. Martens, and L. De Marez, "Qoe measurement of mobile youtube video streaming," in Proceedings of the 3rd workshop on Mobile video delivery, ser. MoViD '10. New York, NY, USA: ACM, 2010, pp. $27-32$. [Online]. Available: http://ᄀdoi.acm.org/ $\neg 10.1145 / \neg 1878022.1878030$

[7] S.-Y. Yang, D. liang Lee, and K.-Y. Chen, "A new ubiquitous information agent system for cloud computing - example on gps and bluetooth techniques in google android platform," in Electric Information and Control Engineering (ICEICE), 2011 International Conference on, 2011, pp. 19291932.

[8] R. Lowe, P. Mandl, and M. Weber, "Context directory: A context-aware service for mobile contextaware computing applications by the example of google android," in Pervasive Computing and 
International Journal of Software Engineering \& Applications (IJSEA), Vol.5, No.4, July 2014

Communications Workshops (PERCOM Workshops), 2012 IEEE International Conference on, 2012, pp. 76-81.

[9] N. Husted, H. Sadi, and A. Gehani, "Smartphone security limitations: conflicting traditions," in Proceedings of the 2011 Workshop on Governance of Technology, Information, and Policies, ser. GTIP '11. New York, NY, USA: ACM, 2011, pp. 5-12. [Online]. Available:

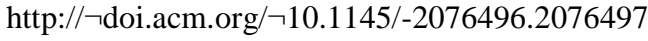

[10] A. Shabtai, Y. Fledel, U. Kanonov, Y. Elovici, S. Dolev, and C. Glezer, "Google android: A comprehensive security assessment," Security Privacy, IEEE, vol. 8, no. 2, pp. 35-44, 2010.

[11] S. Oligny, J.-M. Desharnais, and A. Abran, "A method for measuring the functional size of embedded software," in 3rd International Conference on Industrial Automation, 1999, pp. 7-9.

[12] B. Boehm, R. Valerdi, J. Lane, and A. Brown, "Cocomo suite methodology and evolution," CrossTalk, vol. 18, no. 4, pp. 20-25, 2005.

[13] C. Jones and T. C. Jones, Estimating software costs. McGraw-Hill New York, 1998, vol. 3.

[14] C. Symons, "Come back function point analysis (modernized)-all is forgiven!)," in Proc. of the 4th European Conference on Software Measurement and ICT Control, FESMA-DASMA, 2001, pp. 413426.

[15] M. Lother and R. Dumke, "Points metrics-comparison and analysis," in International Workshop on Software Measurement (IWSM 2001), Montréal, Québec, 2001, pp. 155-172.

[16] J. Engelhart, P. Langbroek et al., Function Point Analysis (FPA) for Software Enhancement. NESMA, 2001.

[17] D. J. Reifer, "Asset-r: A function point sizing tool for scientific and real-time systems," Journal of Systems and Software, vol. 11, no. 3, pp. 159-171, 1990.

[18] M. Maya, A. Abran, S. Oligny, D. St-Pierre, and J.-M. Desharnais, "Measuring the functional size of real-time software," in Proc. of 1998 European Software Control and Metrics Conference, Maastricht, The Netherlands, 1998, pp. 191-199.

[19] S. Kusumoto, F. Matukawa, K. Inoue, S. Hanabusa, and Y. Maegawa, "Estimating effort by use case points: method, tool and case study," in Software Metrics, 2004. Proceedings. 10th International Symposium on, 2004, pp. 292-299.

[20] R. Banker, R. Kauffman, C. Wright, and D. Zweig, "Automating output size and reuse metrics in a repository-based computer-aided software engineering (case) environment," Software Engineering, IEEE Transactions on, vol. 20, no. 3, pp. 169-187, 1994.

[21] J. Matson, B. Barrett, and J. Mellichamp, "Software development cost estimation using function points," Software Engineering, IEEE Transactions on, vol. 20, no. 4, pp. 275-287, 1994.

[22] R. Meli, "Early and extended function point: a new method for function points estimation," in Proceedings of the IFPUG-Fall Conference, 1997, pp. 15-19.

[23] M. Morisio, I. Stamelos, V. Spahos, and D. Romano, "Measuring functionality and productivity in web-based applications: a case study," in Software Metrics Symposium, 1999. Proceedings. Sixth International, 1999, pp. 111-118.

[24] G. Caldiera, G. Antoniol, R. Fiutem, and C. Lokan, "Definition and experimental evaluation of function points for object-oriented systems," in Software Metrics Symposium, 1998. Metrics 1998. Proceedings. Fifth International, 1998, pp. 167-178.

[25] C.-C. S. M. I. Consortium et al., "The cosmic functional size measurement method-version 3.0 measurement manual (the cosmic implementation guide for iso/iec 19761: 2003)," 2007.

[26] R. Meli, A. Abran, V. T. Ho, and S. Oligny, "On the applicability of cosmic-ffp for measuring software throughout its life cycle," in Proceedings of the 11th European Software Control and Metrics Conference, 2000, pp. 18-20.

[27] J. Kammelar, "A sizing approach for oo-environments," in Proceedings of the 4th International ECOOP Workshop on Quantitative Approaches in Object-Oriented Software Engineering, 2000.

[28] S. Abrahão, G. Poels, and O. Pastor, "A functional size measurement method for object-oriented conceptual schemas: design and evaluation issues," Software \& Systems Modeling, vol. 5, no. 1, pp. 48-71, 2006.

[29] P. Forselius, "Finnish software measurement association (fisma), fsm working group: Fisma functional size measurement method v. 1.1." 2004.

[30] B. Kitchenham, "Procedures for performing systematic reviews," Keele, UK, Keele University, vol. 33, p. 2004, 2004.

[31] J.-H. Sohn, J.-H. Woo, M.-W. Lee, H.-J. Kim, R. Woo, and H.-J. Yoo, “A 50 mvertices/s graphics processor with fixed-point programmable vertex shader for mobile applications," in Solid-State 
International Journal of Software Engineering \& Applications (IJSEA), Vol.5, No.4, July 2014

Circuits Conference, 2005. Digest of Technical Papers. ISSCC. 2005 IEEE International, 2005, pp. 192-592 Vol. 1, google.

[32] H. Mukhtar, D. Belad, and G. Bernard, "A model for resource specification in mobile services," in Proceedings of the 3rd international workshop on Services integration in pervasive environments, ser. SIPE '08. New York, NY, USA: ACM, 2008, pp. 37-42, aCM. [Online]. Available:

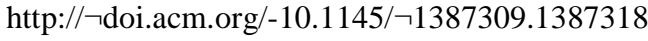

[33] H. Feng, "A literature analysis on the adoption of mobile commerce," in Grey Systems and Intelligent Services, 2009. GSIS 2009. IEEE International Conference on, 2009, pp. 1353-1358, iEEE.

[34]A. Kumar Maji, K. Hao, S. Sultana, and S. Bagchi, "Characterizing failures in mobile oses: A case study with android and symbian," in Software Reliability Engineering (ISSRE), 2010 IEEE 21st International Symposium on, 2010, pp. 249-258, iEEE.

[35] J. Al-Jaroodi, A. Al-Dhaheri, F. Al-Abdouli, and N. Mohamed, "A survey of security middleware for pervasive and ubiquitous systems," in Network-Based Information Systems, 2009. NBIS '09. International Conference on, 2009, pp. 188-193, iEEE.

[36] K. Hameed et al., "Mobile applications and systems," 2010, google.

[37] I. R. D. E. A. G. Alekhya Mandadi, Deepti Mudegowder, "Mobile applications: Characteristics \& group project summary," Mobile Application Development, 2009, google.

[38] M. Hayenga, C. Sudanthi, M. Ghosh, P. Ramrakhyani, and N. Paver, "Accurate system-level performance modeling and workload characterization for mobile internet devices," in Proceedings of the 9th workshop on MEmory performance: DEaling with Applications, systems and architecture, ser. MEDEA '08. New York, NY, USA: ACM, 2008, pp. 54-60, aCM. [Online]. Available:

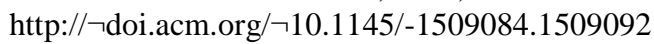

[39] A. Giessmann, K. Stanoevska-Slabeva, and B. de Visser, "Mobile enterprise applications-current state and future directions," in System Science (HICSS), 2012 45th Hawaii International Conference on, 2012, pp. 1363-1372, google.

[40] C. Gencel, R. Heldal, and K. Lind, "On the conversion between the sizes of software products in the life cycle."

[41] F. S. M. A. FiSMA. (2004) Fisma functional size measurement method version 1-1. [Online].

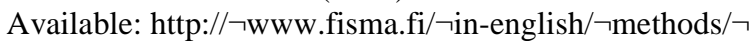

\section{Authors}

Laudson Silva de Souza is masters student the Department of Informatics and Applied Mathematics, Federal University of Rio Grande do Norte, Brazil.

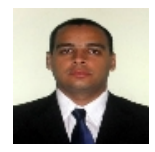

Gibeon Soares de Aquino Jr. is PhD teacher the Department of Informatics and Applied Mathematics, Federal University of Rio Grande do NorteBrazil. 